\title{
Efficacité technique des exploitations avicoles productrices d'œufs au sud du Bénin
}

\author{
Emile Blaise Siéwé Pougoué ${ }^{1}{ }^{*}$ Ibrahim Manu $^{2}$ \\ Innocent Labiyi Adédédji ${ }^{3}$ Thiburce Bokossa ${ }^{4}$
}

\begin{abstract}
Mots-clés
Volaille, poule pondeuse, efficacité, analyse stochastique, conduite d'élevage, Bénin
\end{abstract}

Submitted: 9 November 2017

Accepted: 19 March 2019

Published: 20 May 2019

DOI: $10.19182 /$ remvt. 31728

\section{Résumé}

Dans un contexte d'importation massive de produits avicoles au Bénin, la problématique relative à la performance des exploitations avicoles nationales est au cœur du débat. Afin d'assurer une meilleure allocation des ressources productives, des considérations d'ordre socioéconomiques entrent en jeu. Cette étude visait à établir le niveau de performance des exploitations de poules pondeuses au sud du Bénin. A travers l'approche paramétrique, les niveaux d'efficacité technique ont été estimés à partir d'une frontière stochastique de production. Les données ont été collectées auprès de quarante-cinq exploitations avicoles au moyen d'enquêtes réalisées entre août et septembre 2016. Les analyses ont montré que ces exploitations étaient en majorité performantes malgré des écarts d'efficacité entre elles. Le capital humain, le travail, les traitements vétérinaires étaient les principaux facteurs significatifs du processus de production d'œufs. La régression de la fonction de production a révélé que la faible production résultait davantage de l'insuffisance technique des producteurs (84\%) que de la répartition inefficace des ressources (16\%). Les écarts d'efficacité s'expliquaient par des facteurs socioéconomiques, notamment l'appui de l'Etat, le niveau d'instruction, I'âge de l'aviculteur, ses compétences et la densité des élevages. En conclusion, au Bénin les exploitations avicoles enquêtées étaient performantes mais restaient fragilisées par des externalités.

- Comment citer cet article : Siéwé Pougoué E.B., Manu I., Labiyi Adédédji I., Bokossa T., 2019. Technical efficacy of laying hen farms in Southern Benin. Rev. Elev. Med. Vet. Pays Trop., 72 (1) : 23-32, doi : 10.19182/remvt.31728

\section{INTRODUCTION}

Au Bénin, deux types d'aviculture cohabitent : l'aviculture traditionnelle et l'aviculture commerciale (Guézodje, 2009 ; FAO, 2015). La poule pondeuse et le poulet de chair sont les volailles les plus élevées dans le pays, suivies de la pintade, du canard, du dindon et des pigeons (FAO, 2015). La filière avicole est caractérisée par la prédominance d'exploitations agricoles de petite taille avec une très faible

\footnotetext{
1. Département d'Agriculture, d'élevage et des produits dérivés (AGEPD), Ecole nationale supérieure polytechnique de Maroua (ENSPM), Université de Maroua (UMa), BP 46, Maroua, Cameroun.

2. Faculté d'agronomie et des sciences agricoles, Université de Dschang (FASA-Uds), Département de vulgarisation agricole et sociologie rurale, Dschang, Cameroun. 3. Laboratoire d'analyses et de recherches sur les dynamiques économiques et sociales (Lardes), Département d'économie et de sociologie rurales, Faculté d'agronomie, Université de Parakou, Bénin.

4. Faculté des sciences agronomiques (FSA), Université d'Abomey-Calavi (UAC), Bénin.

* Auteur pour la correspondance

Tél. : +23769792 2212 ; email : siewe_blaise@yahoo.frs
}

capacité d'accès au financement, un faible niveau de biosécurité, et les oiseaux / produits sont vendus aux marchés de volailles vivantes. La majorité de ces exploitations fonctionne de manière informelle, c'està-dire sans suivre de procédure préalable ni les règles en place. Elles se distinguent par leur débrouillardise et une lutte permanente pour leur survie. Les revenus faibles et la force de travail partiellement valorisée rendent peu compétitifs les produits avicoles (MAEP, 2010).

Les maladies comme la grippe aviaire hautement pathogène et autres épizooties constituent l'une des plus fortes contraintes de la filière (Fanou, 2006), suivies de la relative indisponibilité des semences (poussins) (Batonon-Alavo et al., 2015). L'émergence et la persistance des épizooties rendent les petits producteurs particulièrement vulnérables (Guézodje, 2009). Adebowale et Adeyemo (2018) soulignent que les maladies causées par des pathogènes intestinaux influent sur la productivité des pondeuses dans les fermes avicoles. Les épizooties peuvent par ailleurs être responsables de zoonoses. Les impacts socioculturels et économiques directs et indirects de ces maladies influencent les décisions politiques et commerciales, perturbent le marché et causent des pertes économiques.

D’autres facteurs ont été identifiés par Batonon-Alavo et al. (2015) comme la fluctuation des prix et la faible disponibilité des matières 
premières sur les marchés domestiques pour l'alimentation animale. La grande majorité des aviculteurs se contente de produire les aliments dans de petits ateliers artisanaux ; la composition des aliments reste le secret du fabricant et les institutions n'effectuent ni contrôle ni analyse bromatologique, pour guider l'éleveur dans le choix des aliments et la formulation des rations (Nepad et FAO, 2005). Dans ce contexte Batonon-Alavo et al. (2015) soulignent que les vendeurs de matières premières ont des difficultés pour approvisionner tous les éleveurs. Ces derniers admettent que les couvoirs locaux et les fournisseurs de poussins ne disposent pas d'une capacité de production pouvant suffire à la demande en poussins.

En outre, le nombre d'intervenants au sein de la filière peut réduire considérablement les revenus obtenus des aviculteurs. Au Bénin la mercuriale des denrées alimentaires mentionne que le prix d'un œuf dans la commune d'Abomey-Calavi est de 100 francs CFA (0,153 euro), prix nettement au-dessus de la moyenne dans le département de l'Atlantique (96 FCFA soit $0,147 €$ ) et au niveau national (90 FCFA soit $0,137 €$ ) (MAEP, 2014). Or le prix pratiqué à la ferme est généralement de 50 FCFA $(0,076 €)$ montrant une grande différence de prix qui pourrait décourager les éleveurs.

La présence de pratiques informelles côtoyant des activités formelles est l'une des caractéristiques de la filière œufs au Bénin. L'exportation illégale des matières premières végétales vers le Nigeria ou encore la commercialisation des œufs au niveau de la frontière bénino-nigériane témoigne de la présence d'un secteur informel qui peut fragiliser l'organisation en amont et en aval de la filière (Batonon-Alavo et al., 2015). Cette situation pourrait influencer le niveau de performance des exploitations de poules pondeuses au Bénin dans la mesure où elle créerait un déficit de l'offre nationale (par rapport à la demande) en matières premières végétales entrant dans la composition de l'aliment pour volaille.

Très peu d'informations existent sur les niveaux d'efficacité des exploitations avicoles au Bénin. Des études ont été réalisées sur la filière avicole béninoise mais elles n'ont pas estimé les niveaux d'efficacité des exploitations avicoles (Fanou, 2006 ; FAO, 2015 ; Sodjinou et al., 2009 ;

๗ี Chrysostome et Sodjinou, 2005). En revanche, ces niveaux de performance ont été déterminés dans des études portant sur d'autres sujets relatifs à l'agriculture : l'ananas (Kpenavoun Chogou et al., 2017), le soja (Labiyi et al., 2014) ou le riz (Chemak et Dhehibi, 2010).

Ainsi, l'objectif de ce travail a été d'analyser le niveau de performance des exploitations de poules pondeuses, d'une part sur le plan technique, d'autre part en fonction des déterminants sociaux économiques qui sous-tendent la production d'œufs dans la commune d'Abomey-Calavi au sud du Bénin. Cette étude partait de l'hypothèse qu'au sud du Bénin les exploitations de poules pondeuses avaient un niveau de performance technique faible qui était dû a) en majorité à l'inefficacité des producteurs plutôt qu'aux externalités et/ou b) à des facteurs socioéconomiques à la fois endogènes et exogènes à l'exploitation avicole.

\section{MATERIEL ET METHODES}

\section{Concept d'efficacité}

Le concept efficacité fait référence à l'optimum de Pareto (Chemak et Dhéhibi, 2010), qui est une notion minimale de mesure d'efficacité. Cette dernière permet dans certains cas de donner une indication sur la direction générale à prendre ou d'éviter de grossières erreurs de décision. Koopmans propose une définition de l'efficacité dans une logique parétienne appelée « efficacité Pareto-Koopmans » (Thanassoulis, 2001).

La notion d'efficacité consiste pour une entreprise à identifier les besoins de ses clients et à y répondre précisément par son offre de produits ou de services, mettant en œuvre ses compétences pour les réaliser. Elle fait référence au niveau de réalisation d'une activité souhaitée.
C'est une notion relative dans la mesure où c'est dans un groupe défini qu'une unité est efficace, en dehors de ce groupe, elle peut ne plus l'être (Kpenavoun Chogou et al., 2017). En revanche, pour Amara et Romain (2000), l'inefficacité signifie que la capacité optimale visée par l'efficacité est inatteignable.

Au sein d'une unité de production (entreprise, exploitation avicole) trois types d'efficacité sont distingués : l'efficacité technique qui renvoie à l'atteinte de l'objectif de l'entreprise suivant les quantités produites, l'efficacité allocative qui intègre la notion de coût des quantités produites, et l'efficacité économique qui est le produit des deux premiers (Farell, 1957 ; Débreu, 1951 ; Coelli et al., 1998). Une entreprise qui est techniquement et allocativement efficace est par conséquent économiquement efficace. Notre travail a porté sur l'efficacité technique.

Farrell (1957) a défini l'efficacité d'une manière plus explicite en dissociant ce qui est d'origine technique de ce qui est un mauvais choix par rapport au prix des intrants. L'efficacité technique proche du coefficient d'utilisation des ressources de Debreu (1951) relève de la gestion technique des ressources. Atkinson et Cornwell (1994) considèrent qu'une unité de production est techniquement efficace si, à partir du panier d'intrants qu'elle détient, elle produit le maximum d'outputs possible ou si, pour produire une quantité donnée d'outputs, elle utilise les plus petites quantités possible d'intrants.

De façon opérationnelle, une exploitation avicole sera techniquement efficace si elle maitrise au mieux les aspects techniques de la production : les mesures de biosécurité, les aspects bromatologiques et les normes de distribution d'aliments, les aspects prophylactiques ou autres, indispensables pour obtenir une production optimale avec un minimum d'intrants. Une exploitation est performante si elle a la capacité de produire des quantités optimales d'outputs suite à la combinaison optimale d'intrants, la difficulté étant de mesurer cette efficacité.

La littérature spécialisée et les mesures empiriques de l'efficacité technique (Coelli, 1996 ; Amara et Romain, 2000 ; Ambapour, 2001) font remonter sa conceptualisation aux travaux pionniers de Debreu (1951), Koopmans (1951) et surtout Farrell (1957) qui a proposé une approche pour l'estimation des frontières d'efficacité. Les méthodes d'estimation de la frontière peuvent être classées selon « la forme prévue de la frontière, » « la technique d'estimation » utilisée pour l'obtenir, et « la nature et les propriétés supposées de l'écart » entre la production observée et la production optimale (Albouchi et al., 2007). La présentation est centrée sur les méthodes selon la forme prévue de la frontière et celles basées sur les écarts de production.

La nature des écarts entre la production observée et la production maximale différencie les frontières stochastiques et déterministes. En effet, si les écarts sont supposés expliquer uniquement l'inefficacité du producteur, la frontière est dite de nature déterministe ; si les écarts sont supposés expliquer simultanément l'inefficacité du producteur et les éléments aléatoires qui ne dépendent pas de ce dernier, la frontière est dite de nature stochastique (Albouchi et al., 2007). Le terme d'erreur est alors dupliqué afin de préciser davantage les origines de l'inefficacité. Suivant la forme prévue de la fonction, on distingue les approches paramétriques et non paramétriques. Elles diffèrent essentiellement par les hypothèses concernant les résidus. Une frontière de production sera dite paramétrique si l'on impose une forme fonctionnelle (par exemple Cobb-Douglas, Translog) comprenant les éléments déterministe et stochastique (Albouchi et al., 2007). Les approches non paramétriques imposent moins de structure à la frontière mais supposent l'absence d'erreurs aléatoires.

\section{Notion de compétence}

La notion de compétence est fondamentale dans le cadre de la réalisation d'une activité à l'instar de celle relative à la production d'œufs. La compétence correspond à la mobilisation dans l'action d'un certain 
nombre de savoirs combinés de façon spécifique en fonction du cadre de perception que se construit l'auteur de la situation (Wittorski, 1998). Elle permet d'aboutir à une performance, laquelle pour certains ne représente que sa part observable voire mesurable.

Le Boterf (2004) distingue « être compétent » et « avoir des compétences », les deux sont liés. On peut a priori avoir des compétences sans toutefois être compétent. L'utilisation à bon escient du savoir détenu par un individu permet de dire de lui qu'il est compétent. Il doit le valoriser au profit de son activité afin que ses objectifs soient atteints. Pour Le Clainche (2008) la compétence découle de la mise en œuvre de savoirs, de capacités et d'attitudes qui, selon le niveau atteint, assurent une maîtrise plus ou moins importante de la compétence ; on parle alors de « niveaux de compétence ».

Fellows et al. (1997, in Atkinton et al., 2005), dans le cadre de la gestion des petites entreprises agroalimentaires, ont élaboré un modèle servant à quantifier les qualités et compétences d'un gestionnaire. Plus le score est élevé, plus une personne aurait les qualités pour être un bon entrepreneur (un score de 35 peut être considéré comme positif). La performance d'une exploitation dépend du degré de maîtrise de nombreux paramètres tant internes qu'externes. Cette maitrise provient des compétences des employés ou du gestionnaire.

Dans notre étude, cette variable a été introduite afin de mesurer son influence sur la performance, voire l'efficacité des exploitations avicoles. Sur la base du modèle d'Atkinton et al. (2005), une échelle des niveaux de compétence d'un gestionnaire a été conçue (figure 1) avec six niveaux allant de très faible à excellent. Par ailleurs, à la place de la compétence du gestionnaire nous avons parfois utilisé le concept de capital humain.

\section{Echantillonnage et collecte des données}

La collecte des données s'est faite dans des exploitations de poules pondeuses dans la commune d'Abomey-Calavi au sud du Bénin. Leéchantillon a été obtenu par la méthode boule-de-neige dans neuf arrondissements (Calavi, Ouédo, Akassato, Agori, Hêvié, Golo, Godomey, Togba,Zinvié). Cette méthode propose d'échantillonner une population, d'abord par un tirage aléatoire au sein d'une population cible, puis on demande à chaque individu sélectionné d'inclure k personnes dans l'enquête (Wilhelm, 2014). L'objectif est d'augmenter la taille d'un échantillon et constitue une alternative intéressante aux méthodes classiques dès lors qu'il s'agit de trouver des individus ayant des caractéristiques particulières (Wilhelm, 2014) comme élever des poules pondeuses. L'inconsistance d'un répertoire d'aviculteurs (en particulier ceux produisant les œufs de consommation) préalablement identifiés au niveau national, voire local, a justifié en grande partie le choix de cette méthode. Il a été établi que les aviculteurs se connaissaient et constituaient un réseau solide (Union nationale des aviculteurs professionnels, UNAP-Bénin). Ainsi, avec la méthode boule-de-neige la poignée d'exploitations obtenues auprès de l'UNAP-Bénin (10) et du Centre agricole régional pour le développement rural (Carder) (11) ont permis d'identifier les autres.

L'enquête a été réalisée en août et septembre 2016. Les données collectées étaient de sources primaire (questionnaires) et secondaire (documentation). Elles étaient quantitatives (quantité d'aliment, cheptel, superficie des bâtiments, quantité d'œufs pondus, prix de l'aliment, prix de l'alvéole d'œufs, etc.) et qualitatives (souche, origine des poussins, présence/absence de pédiluve, mode d'élevage, type de bâtiment, etc.).

L'unité statistique a été l'exploitation avicole, et l'unité répondante a été le propriétaire et/ou gestionnaire de l'exploitation. La population d'exploitations avicoles de la zone d'étude est estimée à 110 sur lesquelles 85 sont encore en activité (FAO, 2015). Quarante-cinq fermes ont été enquêtées.

Le questionnaire était constitué de deux parties : l'une réservée au propriétaire de l'exploitation, l'autre à son gestionnaire. Celle du propriétaire comprenait les informations relatives à l'identification de la ferme, aux caractéristiques du système d'élevage (type de bâtiment et lieu d'implantation, origine et souches des poussins, niveau de contact des poules pondeuses avec les autres espèces, prophylaxie, biosécurité, approvisionnement en intrants, matériels et équipements, etc.), aux facteurs liés à la production (nature et provenance de l'aliment, nombre de distributions d'aliment et d'eau, ramassage d'œufs, etc.), aux paramètres économiques, au soutien de l'Etat, et autres. Celle du gestionnaire contenait l'évaluation de la compétence, le suivi des activités quotidiennes de la ferme, des pontes, des aspects sanitaires, etc. En l'absence du propriétaire c'était le gestionnaire qui répondait à l'ensemble du questionnaire et vice-versa. Outre les entretiens réalisés sur une base déclarative, les documents de suivis technico-économiques de la ferme (production, commercialisation, entre autres) ont été exploités afin de vérifier la cohérence des données issues des entretiens. Certaines informations (pédiluve, type de bâtiment) ont été obtenues à travers des observations. La productivité des poules pondeuses telle qu'elle était déterminée dans la fonction de production a été estimée annuellement pour affiner les calculs.

L'analyse des données a été réalisée avec Frontière 4.1 du logiciel $\mathrm{R}$ (version 3.2.3) pour la régression de la fonction de production. Stata a été utilisé pour l'estimation des paramètres du modèle Tobit relatif à l'analyse des déterminants de la performance.

\section{Spécification du modèle empirique d'analyse}

Etant donné le caractère aléatoire de la production avicole au Bénin, et particulièrement dans la région Sud, lié aux fluctuations du prix des facteurs de production et aux risques sanitaires, le choix de la méthode paramétrique stochastique pour estimer l'efficacité était primordial dans cette étude. Pour Ambapour (2001), la forme générale de la fonction de production de l'approche stochastique, proposée simultanément par Aigner et al. (1977), et Meusen et Van Den Broeck (1977), se présente ainsi :

$Y=f(X, \beta)+(v-u)(1)$

$u \geq 0 ;-\infty \leq v \leq+\infty$

De façon détaillée, l'équation (1) peut s'écrire ainsi :

$Y i=\beta o+\sum \beta j X i j+\varepsilon i(2)$

où $\varepsilon i=v i-u i$

Soit la forme matricielle $Y=X \beta+\varepsilon$ (3)

où $Y$ représente la production, $X$ les intrants de production, $\beta$ les paramètres fixes à estimer selon la forme de l'équation (1), $\beta$ l'élasticité de la production par rapport à l'intrant $X$, et $u_{i}$ les valeurs positives d'une

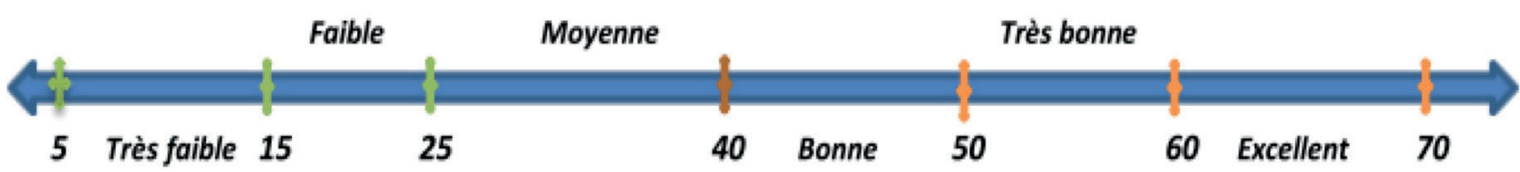

Figure 1 : schématisation des différents niveaux de compétence d'un exploitant dans les élevages de pondeuses au Sud Bénin. Schéma élaboré à partir de l'échelle construite par Atkinton et al., 2005. 
variable aléatoire à laquelle on associe l'effet d'inefficacité technique du producteur $i$. Les termes d'erreur $v$ sont associés aux erreurs de mesure et autres facteurs aléatoires, comme le climat, le vol et les conflits de voisinage, pouvant influencer la production.

Selon Panda (1996), les $v_{i}$ ont une distribution normale, dont la moyenne $\mu_{v}$ est égale à 0 et la variance $\sigma_{v}{ }^{2}$ est une constante, et sont indépendants des $u_{i}$, qui sont supposés avoir une distribution semi-normale avec aussi une moyenne $\mu_{u}=0$ et une variance constante $\sigma_{u}{ }^{2}$.

Dans ces conditions, le ratio de la valeur observée de l'output du i ${ }^{e}$ producteur par rapport à l'output potentiel défini par la frontière de production (compte tenu des intrants de production dans l'exploitation $\mathrm{X}_{i}$ ) est utilisé pour calculer son efficacité technique $\left(\mathrm{ET}_{\mathrm{j}}\right)$ :

$$
E T_{i}=\frac{Y i}{\exp \left(\beta \sum \ln x i\right)}=\frac{\left.\exp \left(\beta \sum \ln x i\right)-u i\right)}{\exp \left(\beta \sum \ln x i\right)}=\exp (-u i)
$$

où $\exp ($.) représente la fonction exponentielle.

La transposition du modèle (2) est la suivante :

$$
\begin{aligned}
\operatorname{Ln}\left(Q_{i}\right)= & \operatorname{Ln}\left(\beta_{0}\right)+\beta_{1} \operatorname{Ln}\left(X_{1 i}\right)+\beta_{2} \operatorname{Ln}\left(X_{2 i}\right)+\beta_{3} \operatorname{Ln}\left(X_{3 i}\right)+ \\
& \beta_{4} \operatorname{Ln}\left(X_{4 i}\right)+\beta_{5} \operatorname{Ln}\left(X_{5 i}\right)+\beta_{6} \operatorname{Ln}\left(X_{6 i}\right)+\varepsilon_{i}
\end{aligned}
$$

où $i$ représente l'exploitation, $Q_{i}$ la quantité d'œufs pondus par poule par an dans l'exploitation $i, X_{1 i}$ la superficie des bâtiments $\left(\mathrm{m}^{2}\right)$ exploitée dans l'exploitation $i, X_{2 i}$ la compétence du gestionnaire (capital humain) dans l'exploitation $i, X_{3 i}$ la quantité d'aliments distribués avant la ponte (18 $-19^{\mathrm{e}}$ semaine) dans l'exploitation $i, X_{4 i}$ les dépenses relatives au traitement vétérinaire (FCFA/poule/cycle) dans l'exploitation $i, X_{5 i}$ le travail alloué (h/pondeuse/cycle) dans l'exploitation $i ; X_{6 i}$ la dépense par poule prête à pondre dans l'exploitation $i . \beta_{0} \beta_{1} \beta_{2} \beta_{3} \beta_{4} \beta_{5} \beta_{6}$ représentent respectivement les élasticités liées à chacune des variables. L'existence des inefficacités a été vérifiée avec la procédure adoptée par Coelli (1996), qui consiste à maximiser le logarithme népérien de la fonction de vraisemblance et à calculer le ratio de vraisemblance.

\section{Régression des scores d'efficacité : déterminants de l'efficacité}

L'explication des inefficacités se déroule en deux étapes (Coeilli, 1998 ; Labiyi et al., 2014) : la première permet d'estimer les inefficacités à partir d'une fonction de production. La deuxième aboutit sur une régression des scores d'efficacité dans l'optique de déterminer les facteurs qui influent sur l'efficacité technique des aviculteurs. Pour ces modèles, la variable dépendante (ou variable expliquée) est continue mais n'est observable que sur un certain intervalle. Ces modèles sont également qualifiés de modèles de régression censurée ou modèles de régression tronquée. Le choix du modèle Tobit (Albouchi et al., 2007 ; Labiyi et al., 2014) était ici justifié par le fait que les indices d'efficacité étaient continus et prenaient des valeurs comprises entre 0 et 1 . La relation suivante est relative à la régression des scores d'ET :

$\mathrm{ET}_{\mathrm{i}}=\mathrm{a}_{\mathrm{o}}+\sum_{j=1}^{n} a_{i j} X_{i j}^{\prime}+u_{i}(6)$

où $i$ représente le numéro de l'exploitation avicole et $j$ celui de la variable considérée, facteur pouvant influencer la performance. Le tableau I donne des indications sur la dénomination, les modalités et les signes attendus des différentes variables.

\section{RESULTATS ET DISCUSSION}

\section{Estimation de l'efficacité des exploitations avicoles productrices d'oufs}

Les statistiques descriptives (tableau II) des variables de la fonction de production indiquent que chaque exploitation occupait en moyenne 212 mètres carrés de bâtiment. La productivité annuelle des pondeuses était en moyenne de $238( \pm 27)$ œufs. Les traitements vétérinaires étaient évalués à 448 FCFA/poule/cycle. Le score de compétence de 42,88 a été jugé bon (Atkinton et al., 2005).

Les résultats de l'estimation du modèle stochastique (tableau III) montrent que tous les paramètres des variables de la fonction de production étaient positifs. Ils confirment la relation positive attendue entre ces facteurs de production et le produit de ces exploitations (Chemak et Dehibi, 2010).

La valeur du rapport de variance $\gamma(0,8375437)$, significativement différente de zéro à $0,1 \%$, indique l'existence des inefficacités productives. Environ $84 \%$ des écarts entre la productivité observée et la productivité potentielle des pondeuses étaient dus à l'inefficacité des aviculteurs. En effet, $16 \%$ des écarts étaient liés à des effets aléatoires, y compris des erreurs de mesures, ce qui pouvait provenir de la nature des données. Plus la valeur de $\gamma$ se rapproche de 1, plus la différence entre les résultats issus d'une estimation stochastique et ceux issus d'une estimation déterministe est faible (Albouchi et al., 2007).

Des six intrants introduits dans le modèle stochastique de production (tableau III), les coefficients de trois d'entre eux ont été positivement significatifs, notamment la compétence des gestionnaires (capital humain), le temps de travail et les traitements vétérinaires. L'estimation des élasticités partielles de production par rapport à ces facteurs révèle l'impact (Chemak et Déhibi, 2010) de la compétence des gestionnaires sur la production. Les coefficients relatifs à chaque variable du modèle représentent leur élasticité respective. Lélasticité de la production des œufs en capital humain (compétence) a été de 0,3856 , celle du travail de 0,0823 et celle des soins vétérinaires de 0,0738 , soit une augmentation respective de $0,39 \%, 0,08 \%$ et $0,07 \%$ de la production des pondeuses pour $1 \%$ d'augmentation des variables considérées.

Les scores d'efficacité technique (ET) ont été estimés en moyenne à 92,38 \% (tableau IV). Globalement, 68,89\% des fermes enquêtées ont eu un score d'efficacité supérieur ou égal à la moyenne. Les quartiles d'ordre 1,2 (médiane $=93,3 \%$ ) et 3 montrent que $25 \%, 50 \%$ et $75 \%$ des scores d'efficacité étaient respectivement inférieurs ou égaux à 90,23\%, 93,37\% et 95,20\%. Ainsi, les aviculteurs étaient en majorité techniquement efficaces (tableau V). Néanmoins, il était possible d'améliorer ces niveaux d'efficacité $\left(\mathrm{ET}_{\min }=68,65 \%\right.$ et $\left.\mathrm{ET}_{\max }=98,10 \%\right)$.

Estimé à 29,45 \% de la valeur absolue des scores d'efficacité extrêmes (98,1\%-68,65\%), cet écart représente 30,02\% $(29,45 / 98,10)$ de l'efficacité maximale. En considérant les extrêmes, la différence d'ET entre les fermes traduit les marges de progrès possibles. Atteindre le niveau d'efficacité de la ferme la plus efficace de l'échantillon permettrait à une ferme d'économiser jusqu'à cette valeur de la ressource gaspillée. Toutefois, la préoccupation centrale résidait autour des déterminants de l'ET.

Nos résultats correspondent à ceux obtenus par certains auteurs ayant abordé la performance des producteurs via les analyses d'efficacité. Labiyi et al. (2014) rapportent une moyenne des indices d'ET de 0,640 chez les producteurs de soja au Bénin et soulignent que l'amélioration du niveau d'ET de production nécessite des actions ciblées. Egalement au Bénin, Kpenavoun Chogou et al. (2017) montrent que l'ET moyenne des producteurs d'ananas de la variété Pain de Sucre est significativement inférieure à celle des producteurs de la variété Cayenne Lisse. Albouchi et al. (2007), dans leurs travaux sur les zones irriguées de Tunisie centrale, montrent qu'il existe un différentiel d'ET entre les zones étudiées avec des scores variant entre $96 \%$ et $64,2 \%$. Si $43 \%$ des écarts entre la production observée et la production potentielle des filières sont liés à des effets aléatoires, 57 \% sont dus à l'inefficacité desdites filières. Sharma et al. (1999), en adoptant les approches paramétriques et non paramétriques, montrent que les producteurs de porcs à Hawaii sont techniquement efficaces dans leurs activités de production porcine. 


\section{Tableau I}

Variables, modalités et signes attendus pour déterminer l'efficacité technique des élevages de pondeuses au Sud Bénin (août-septembre 2016)

\begin{tabular}{|c|c|c|c|}
\hline $\begin{array}{l}\text { Variables } \\
\text { dépendantes }\end{array}$ & Variables indépendantes & Modalités & $\begin{array}{l}\text { Signes } \\
\text { attendu. }\end{array}$ \\
\hline \multirow{25}{*}{$\begin{array}{l}\text { Score d'efficacité } \\
\text { de l'exploitation } \\
\mathrm{i}\left(\mathrm{ET}_{\mathrm{i}}\right)\end{array}$} & Age du propriétaire de l'exploitation $\left(X_{1 i}^{\prime}\right)$ & Variable continue & $+/-$ \\
\hline & Niveau d'instruction du propriétaire ou nb. d'années d'étude $\left(X_{2 \mathrm{i}}^{\prime}\right)$ & Variable continue & + \\
\hline & Niveau d'instruction de l'éleveur $\left(X_{3 i}^{\prime}\right)$ & Variable continue & - \\
\hline & Compétence du gestionnaire de l'exploitation $\left(X_{4 i}^{\prime}\right)$ & Variable continue & $+/-$ \\
\hline & Poids moyen de la poule à l'entrée en ponte $\left(X_{5 i}^{\prime}\right)$ & Variable continue & $+/-$ \\
\hline & Densité des poules / $\mathrm{m}^{2}\left(\mathrm{X}_{6 \mathrm{i}}^{\prime}\right)$ & Variable continue & $+/-$ \\
\hline & Nb. d'années d'expérience $\left(X_{7 \mathrm{i}}^{\prime}\right)$ & Variable continue & + \\
\hline & Dépenses liées à la fidélisation du client $\left(\mathrm{X}_{8 \mathrm{i}}\right)$ & Variable continue & + \\
\hline & Distance de la ferme par rapport aux habitations $\left(X_{9 i}^{\prime}\right)$ & Variable continue & + \\
\hline & Distance de la ferme par rapport à la ferme la plus proche $\left(X^{\prime}{ }_{10 i}\right)$ & Variable continue & + \\
\hline & Présence de pédiluve $\left(X^{\prime}{ }_{11 i}\right)$ & Variable binaire $(0=$ non, $1=$ oui $)$ & + \\
\hline & Contrat avec les fournisseurs d'intrants $\left(X^{\prime}{ }_{12 i}\right)$ & Variable binaire $(0=$ non, $1=$ oui $)$ & + \\
\hline & Appartenance à un groupe $\left(X_{13 \mathrm{i}}^{\prime}\right)$ & Variable binaire $(0=$ non, $1=$ oui $)$ & + \\
\hline & Accès au crédit par le producteur $\left(X^{\prime}{ }_{14 i}\right)$ & Variable binaire $(0=$ non, $1=$ oui $)$ & + \\
\hline & Coût de l'aliment ponte $\left(X_{15 i}^{\prime}\right)$ & Variable continue & - \\
\hline & Prix de vente d'un plateau d'œufs $\left(X^{\prime}{ }_{16 i}\right)$ & Variable continue & + \\
\hline & Appui apporté par les pouvoirs publics à l'aviculteur $\left(X^{\prime}{ }_{17 i}\right)$ & Variable binaire ( 0 = faible, $1=$ grand $)$ & $+/-$ \\
\hline & Nb. de membres actifs dans l'exploitation $\left(X^{\prime}{ }_{18 i}\right)$ & Variable continue & $+/-$ \\
\hline & Sexe du responsable de l'exploitation $\left(X^{\prime}{ }_{19 i}\right)$ & Variable binaire $(0=\mathrm{F}, 1=\mathrm{H})$ & $+/-$ \\
\hline & Statut marital du responsable de l'exploitation $\left(X^{\prime}{ }_{20 i}\right)$ & Variable binaire ( 0 = célibataire, 1 = marié $)$ & $+/-$ \\
\hline & Statut professionnel du responsable de l'exploitation $\left(X_{21}^{\prime}\right)$ & $\begin{array}{l}\text { Variable binaire }(0=\text { à son compte, } \\
1=\text { fonctionnaire/privé) }\end{array}$ & $+/-$ \\
\hline & Fréquence de distribution des aliments $\left(\mathrm{X}_{22 \mathrm{i}}^{\prime}\right)$ & Variable continue & $+/-$ \\
\hline & Rupture du stock d'aliments $\left(X_{23 i}^{\prime}\right)$ & Variable binaire $(0=$ non, 1 = oui $)$ & - \\
\hline & Nature de l'aliment $\left(X_{24 i}^{\prime}\right)$ & $\begin{array}{l}\text { Variable binaire }(0=\text { fabriqué par l'éleveur, } \\
1=\text { achat aliment complet) }\end{array}$ & $+/-$ \\
\hline & Litige avec le voisinage $\left(X^{\prime}{ }_{25 i}\right)$ & Variable binaire $(0=$ non, $1=$ oui $)$ & - \\
\hline
\end{tabular}

\section{Tableau II}

Statistiques descriptives relatives aux variables de la fonction de production pour déterminer I'efficacité technique des élevages de pondeuses au Sud Bénin (août-septembre 2016)

\begin{tabular}{|c|c|c|c|c|}
\hline & Minimum & Maximum & Moyenne & Ecart-type \\
\hline Productivité annuelle / pondeuse & 118,00 & 296 & 238,38 & 27,083 \\
\hline Score de compétence & 20 & 55 & 42,88 & 8,56 \\
\hline Quantité aliment (1-18 semaines) (kg) & 5,35 & 9,51 & 7,32 & 0,92154 \\
\hline Superficie des bâtiments exploités $\left(\mathrm{m}^{2}\right)$ & 36,00 & 1600 & 212,92 & 234,436 \\
\hline Dépense/poule jusqu'à la ponte (FCFA) & 1337,39 & 3500,28 & 2302,80 & 1737,917 \\
\hline Travail alloué (h/poule/cycle) & 3,54 & 19,42 & 10,72 & 4,226 \\
\hline Coûts traitements vétérinaires (FCFA/poule/cycle) & 214,28 & 600 & 448,16 & 106,406 \\
\hline
\end{tabular}




\section{Tableau III}

Estimation de la fonction stochastique de production des élevages de pondeuses au Sud Bénin (août-septembre 2016)

\begin{tabular}{|c|c|c|c|}
\hline Variables ${ }^{1}$ & $\begin{array}{l}\text { Paramètres } \\
\text { estimés }\end{array}$ & $\begin{array}{l}\text { Erreurs } \\
\text { standard }\end{array}$ & $\operatorname{Pr}(>|z|)$ \\
\hline (Intercept) & 3,303 & 0,518 & $1,759 \mathrm{e}-10 * * *$ \\
\hline $\log \left(X_{1}\right)$ & 0,014 & 0,02 & NS \\
\hline $\log \left(X_{2}\right)$ & 0,386 & 0,055 & $3,086 \mathrm{e}-12 * * *$ \\
\hline $\log \left(X_{3}\right)$ & 0,004 & 0,091 & NS \\
\hline $\log \left(X_{4}\right)$ & 0,074 & 0,043 & $0,0827736^{\mu}$ \\
\hline $\log \left(X_{5}\right)$ & 0,082 & 0,03 & $0,0062387 * *$ \\
\hline $\log \left(X_{6}\right)$ & 0,011 & 0,042 & NS \\
\hline \multicolumn{4}{|c|}{ Elasticité partielle de la production } \\
\hline $\mathrm{E}_{\mathrm{Q} / \mathrm{X} 1}$ & 0,014 & - & - \\
\hline $\mathrm{E}_{\mathrm{Q} / \mathrm{X} 2}$ & 0,386 & - & - \\
\hline $\mathrm{E}_{\mathrm{Q} / \mathrm{X} 3}$ & 0,004 & - & - \\
\hline $\mathrm{E}_{\mathrm{Q} / \mathrm{X} 4}$ & 0,074 & - & - \\
\hline $\mathrm{E}_{\mathrm{Q} / \mathrm{X} 5}$ & 0,082 & - & - \\
\hline $\mathrm{E}_{\mathrm{Q} / \mathrm{X} 6}$ & 0,011 & - & - \\
\hline $\begin{array}{l}\text { Economie } \\
\text { d'échelle }\end{array}$ & 0,571 & - & - \\
\hline \multicolumn{4}{|c|}{ Paramètres d'efficience } \\
\hline & 0,011 & 0,005 & $0,0258861 *$ \\
\hline & 0,002 & 0,001 & $0,0888586^{\mu}$ \\
\hline & 0,013 & 0,004 & $0,0019010 * *$ \\
\hline & 0,838 & 0,128 & $6,872 \mathrm{e}-11 * * *$ \\
\hline
\end{tabular}

${ }^{\mu}$ Significatif à $10 \%$; * significatif à $5 \%$; ** significatif à $1 \%$; *** significatif à $0,1 \%$

${ }^{1} \mathrm{X}_{1}$ : superficie des bâtiments par $\mathrm{m}^{2} ; \mathrm{X}_{2}$ : compétence du gestionnaire (capital humain) $; \mathrm{X}_{3}$ : quantité d'aliments distribués avant la ponte $\left(18^{\mathrm{e}}-19^{\mathrm{e}}\right.$ semaine $)$;

$\mathrm{X}_{4}$ : traitement vétérinaire / poule/cycle $(\mathrm{FCFA}) ; \mathrm{X}_{5}$ : travail alloué / poule/cycle (heures) ; $\mathrm{X}_{6}$ : dépense / poule prête à pondre

\section{Tableau IV}

Distribution relative aux scores d'efficacité technique des élevages de pondeuses au Sud Bénin (août-septembre 2016)

\section{Scores d'efficacité Fréquences absolues Fréquences relatives}

(\%)

$\begin{array}{lrc}{[0,70]} & 1 & 2,22 \\ 170,80] & 0 & 0 \\ ] 80,90] & 13 & 28,89 \\ 190,100] & 31 & 68,89 \\ \text { Total } & 45 & 100\end{array}$

Statistiques descriptives des scores d'efficacité technique (\%)

Moyenne

Quartile d'ordre 1

$92,38 \pm 4,84$

Quartile

90,23

Médiane

93,37

Quartile d'ordre 3

95,20

Minimum

68,65

Maximum

98,10

\section{Tableau V}

Test de significativité des scores d'efficacité technique des élevages de pondeuses au Sud Bénin (août-septembre 2016)

\begin{tabular}{|c|c|c|c|c|c|}
\hline & \multicolumn{5}{|c|}{ Valeur du test $=0,5$} \\
\hline & Moyenne & $t$ & ddl & $\begin{array}{c}\text { Sig. } \\
\text { (bilatérale) }\end{array}$ & $\begin{array}{c}\text { Différence } \\
\text { moyenne }\end{array}$ \\
\hline $\begin{array}{l}\text { Efficacité } \\
\text { technique }\end{array}$ & $\begin{array}{c}0,923 \pm \\
0,0484\end{array}$ & 58,711 & 44 & 0,0000 & 0,4238 \\
\hline $\begin{array}{l}\text { Hypothèse } \\
\mathrm{H}_{0} \text { : moyer } \\
\mathrm{H}_{1} \text { : moyer }\end{array}$ & $\begin{array}{l}\text { * } \\
\text { ne (écart-ty } \\
\text { ne (écart-ty }\end{array}$ & $\begin{array}{l}\text { e) }=0,5 \\
\text { e) }>0,5\end{array}$ & & & \\
\hline
\end{tabular}

* Le niveau de performance technique faible était dû : $\mathrm{H}_{0}$ en majorité à l'inefficacité des producteurs plutôt qu'aux externalités ; $\mathrm{H}_{1}$ à des facteurs socioéconomiques à la fois endogènes et exogènes à l'exploitation avicole.

\section{Analyse des déterminants de l'efficacité technique des fermes avicoles}

Le tableau VI montre notamment que téléphone restait le canal le plus utilisé dans la zone d'étude. Chaque aviculteur dépensait en moyenne 2185,56 FCFA/semaine pour le téléphone, leur permettant d'être à

\section{Tableau VI}

Statistiques descriptives des variables quantitatives de la fonction de I'efficacité technique des élevages de pondeuses au Sud Bénin (août-septembre 2016)

\begin{tabular}{|c|c|c|c|c|}
\hline Variables & Min. & Max. & Moy. & ET \\
\hline Compétence & 20 & 55 & 42,88 & 8,56 \\
\hline $\begin{array}{l}\text { Age du propriétaire } \\
\text { de la ferme (années) }\end{array}$ & 29 & 69 & 45,22 & 10,139 \\
\hline $\begin{array}{l}\text { Niveau d'instruction } \\
\text { du propriétaire } \\
\text { (années d'étude) }\end{array}$ & 4 & 20 & 12,50 & 4,413 \\
\hline $\begin{array}{l}\text { Niveau d'instruction } \\
\text { du gestionnaire } \\
\text { (années d'étude) }\end{array}$ & 4 & 20 & 12,41 & 4,19 \\
\hline $\begin{array}{l}\text { Poids moyen de la poule } \\
\text { à l'entrée en ponte }(\mathrm{kg})\end{array}$ & 1,20 & 1,70 & 1,51 & 0,122 \\
\hline Densité (poules/m²) & 2 & 8,30 & 4,9 & 1,538 \\
\hline Années d'expérience & 1 & 23,00 & 7,36 & 6,681 \\
\hline $\begin{array}{l}\text { Dépenses liées à la } \\
\text { fidélisation du client } \\
\text { (FCFA/alvéole) }\end{array}$ & 0 & 200 & 64,11 & 58,146 \\
\hline $\begin{array}{l}\text { Distance ferme- } \\
\text { habitations }(\mathrm{m})\end{array}$ & 5 & 5000 & 694,87 & 1124,241 \\
\hline $\begin{array}{l}\text { Distance ferme-ferme } \\
(\mathrm{m})\end{array}$ & 6 & 10000 & 1503,57 & 1876,833 \\
\hline $\begin{array}{l}\text { Taux de mortalités par } \\
\text { cycle }(\%)\end{array}$ & 0,16 & 23 & 7,86 & 0,05992 \\
\hline $\begin{array}{l}\text { Dépense d'accès } \\
\text { à l'information } \\
\text { (FCFA/semaine) }\end{array}$ & 0 & 10000 & 2185,56 & 2609,82 \\
\hline $\begin{array}{l}\text { Taille de l'exploitation } \\
\text { (membres actifs) }\end{array}$ & 0 & 7 & 3,06 & 1,7372 \\
\hline
\end{tabular}

Min. : minimum ; Max. : maximum ; Moy. : moyenne ; ET : écart-type 
l'écoute des tendances du marché, comme les besoins de la clientèle pour les commandes d'œufs, les échanges avec les fournisseurs par rapport à la disponibilité en aliments ou ingrédients, et leurs prix. Concernant la fidélisation de la clientèle, les fermiers dépensaient en moyenne 64,11 FCFA par alvéole soit environ 2,14 FCFA par œuf (les $3 \mathrm{R}$, réduction, rabais et ristourne, offerts aux grossistes par les aviculteurs).

Les aviculteurs avaient plus de sept ans d'expérience. Le taux de mortalités des volailles $(7,85 \%)$ a été inférieur à $10 \%$ sur toute la période et plus élevé en phase de ponte $(3,38 \%)$ qu'en phase poulette $(2,38 \%)$ et démarrage $(1,79 \%)$. Le stress lié à la manipulation des oiseaux ou leur mauvaise installation par les éleveurs pouvaient expliquer les mortalités au démarrage. Ensuite, l'abandon du pédiluve par de nombreux fermiers (plus de $13 \%$ ) à partir de la vingtième semaine était, parmi d'autres, une des causes des mortalités. Peu d'éleveurs $(46,7 \%)$ adhéraient à une organisation d'aviculteurs (tableau VII).

Le tableau VIII montre le lien significatif des facteurs retenus sur les niveaux d'ET et leur significativité dans les exploitations étudiées. Il existait une corrélation positive entre le nombre d'années d'étude des propriétaires et les niveaux d'efficacité dans l'utilisation des facteurs de production et dans l'allocation de ces facteurs à faible coût. De fait, une année d'étude supplémentaire du responsable de l'exploitation accroissait l'ET de 1,05\%. Plus de $58 \%$ avaient un niveau d'étude supérieur ou égal au baccalauréat et $40 \%$ complétaient leurs études par au moins une formation en aviculture avant leur installation. D'autres auteurs rapportent des résultats semblables. Coelli et Fleming

\section{Tableau VII}

Statistiques descriptives relatives aux variables qualitatives de la fonction de l'efficacité économique des élevages de pondeuses au Sud Bénin (août-septembre 2016)

\begin{tabular}{llc} 
Variables & Oui & $\begin{array}{c}\text { Fréquences } \\
\text { relatives } \mathbf{\%} \text { ) }\end{array}$ \\
\hline $\begin{array}{l}\text { Adhésion à un groupe } \\
\text { d'aviculteurs }\end{array}$ & Non & 46,7 \\
Contrat avec les & Oui & 53,3 \\
fournisseurs & Non & 82,2 \\
Soutien de l'Etat & Oui & 17,8 \\
& Non & 13,3 \\
Litige avec le voisinage & Oui & 86,7 \\
& Non & 80 \\
Présence de pédiluve à & Oui & 20 \\
l'entrée en ponte & Non & 13,3 \\
Accès au crédit & Oui & 86,7 \\
Fréquence journalière & Non & 46,66 \\
d'offre d'aliment & Matin uniquement & 31,11 \\
& Matin, midi et soir & 62,22 \\
Statut social & Fonctionnaire & 6,67 \\
& Salarié privé & 20 \\
Fomme & A son compte & 4,44 \\
& Salarié en retraite & 64,45 \\
& & 11,11 \\
& & 84,44 \\
& & 15,56
\end{tabular}

(2004) montrent que le niveau d'instruction a un impact positif sur l'ET des exploitants. Labiyi et al. (2014) montrent que les indices d'ET augmentent de 0,047 entre un producteur de soja non instruit et un producteur instruit au sud du Bénin. L'influence significative du niveau de compétence des éleveurs $(\mathrm{p}<0,05)$ expliqué par le niveau d'étude, les formations reçues avant l'installation, et les expériences engrangées corrobore les résultats de Fellows et al. (1997).

Les contrats avec les fournisseurs influencent positivement les scores d'ET. Les partenariats économiques entre les aviculteurs, les fournisseurs $(82,2 \%$ ) et les revendeurs d'œufs (53,3 \%) (tableau VII),

\section{Tableau VIII}

Facteurs influençant l'efficacité technique des producteurs de pondeuses au Sud Bénin (août-septembre 2016)

\begin{tabular}{|c|c|c|c|}
\hline Variables & Coefficients & $t$ & $P>|t|$ \\
\hline Age & 0,0038061 & 3,35 & $0,015^{*}$ \\
\hline $\begin{array}{l}\text { Niveau d'instruction du } \\
\text { fermier }\end{array}$ & 0,0105593 & 2,54 & $0,044^{*}$ \\
\hline $\begin{array}{l}\text { Niveau d'instruction de } \\
\text { l'éleveur }\end{array}$ & $-0,0206302$ & $-3,50$ & 0,013* \\
\hline Compétence fermier & 0,0100618 & 5,20 & $0,002 * *$ \\
\hline Poids d'entrée en ponte & $-0,1712156$ & $-2,25$ & $0,065^{\mu}$ \\
\hline Densité & 0,024829 & 2,88 & $0,028^{*}$ \\
\hline $\begin{array}{l}\text { Années d'expérience } \\
\text { du fermier }\end{array}$ & $-0,0025132$ & $-1,74$ & NS \\
\hline Contrat fournisseur & 0,050461 & 2,33 & $0,058^{\mu}$ \\
\hline Adhésion groupe & $-0,0231762$ & $-1,36$ & NS \\
\hline Appui de l'Etat & $-0,1837483$ & $-4,18$ & $0,006^{* *}$ \\
\hline Accès au crédit & 0,005332 & 0,32 & NS \\
\hline Membres actifs & $-0,0271885$ & $-2,58$ & $0,042 *$ \\
\hline Distribution d'aliments & 0,1543722 & 4,39 & $0,005^{* *}$ \\
\hline $\begin{array}{l}\text { Conflit avec le } \\
\text { voisinage }\end{array}$ & $-0,0730765$ & $-2,98$ & $0,025^{*}$ \\
\hline $\begin{array}{l}\text { Dépense de fidélisation } \\
\text { (grossistes) }\end{array}$ & $-0,1178283$ & $-3,79$ & $0,009^{* *}$ \\
\hline $\begin{array}{l}\text { Distance ferme- } \\
\text { habitations }\end{array}$ & 0,0162739 & 3,53 & $0,012^{*}$ \\
\hline Distance ferme-ferme & 0,0297031 & 2,34 & $0,058^{\mu}$ \\
\hline Coût aliment ponte & 0,1470015 & 3,82 & $0,009 * *$ \\
\hline Prix de vente œuf & 0,8054264 & 4,94 & $0,003^{* *}$ \\
\hline Femme & $-0,0956836$ & $-3,37$ & $0,015^{*}$ \\
\hline Fonctionnaire & $-0,1945158$ & $-4,54$ & $0,004^{* *}$ \\
\hline Salarié du privé & 0,1680023 & 4,54 & $0,004^{* *}$ \\
\hline $\begin{array}{l}\text { Achat de l'aliment } \\
\text { complet sur le marché }\end{array}$ & 0,0156438 & 1,01 & NS \\
\hline $\begin{array}{l}\text { Composition de } \\
\text { l'aliment par le fermier }\end{array}$ & 0,0081421 & 0,32 & NS \\
\hline Constante & $-6,636694$ & $-4,41$ & NS \\
\hline \multirow[t]{2}{*}{ /sigma } & 0,0244566 & & \\
\hline & \multicolumn{3}{|c|}{$\begin{array}{l}\text { Log likelihood }=64,92448 \text { LR } \\
\text { chi }^{2}(24)=49,20 \\
\text { Pseudo } R^{2}=0,6101 \text { Prob }> \\
\text { chi }^{2}=0,0018\end{array}$} \\
\hline
\end{tabular}


bien que tacites, résolvent en partie les problèmes de trésorerie à l'origine des ruptures de stock d'aliments et des difficultés d'accès au marché ; ainsi les livraisons peuvent se faire à crédit (œufs et intrants) avec des échéances de paiement différé satisfaisant les deux parties (aucune garantie n'est exigée). Ces contrats informels moraux ont une grande importance dans le fonctionnement de la filière avicole (Batonon-Alavo et al., 2015). Siéwé et al. (2017) rapportent que ce type de partenariat économique entre les fournisseurs et les producteurs de maïs dans la région de l'ouest du Cameroun permet à ces derniers de bénéficier de l'achat d'intrants à crédit, ce qui contribue à améliorer la productivité.

De même, la densité et la fréquence de distribution d'aliments (une, deux ou trois fois par jour) étaient positivement significatives. La densité moyenne de 4,9 volailles par mètre carré enregistrée auprès des éleveurs (tableau VI) était inférieure à la norme de cinq volailles par mètre carré (PACA, 2013). Ainsi, un oiseau supplémentaire par mètre carré de bâtiment contribuait à un accroissement du score d'ET de 0,024 (tableau VIII). En outre, passer d'une à deux distributions par jour contribuait positivement à la productivité des poules : plus de $62 \%$ des aviculteurs alimentaient les poules deux fois par jour et $31 \%$ le faisaient une fois pour des productivités respectives de 241 et 235 œufs par pondeuse (tableau VII). En revanche, la minorité qui distribuait l'aliment trois fois par jour a obtenu un plus faible rendement (230 œufs). Les aviculteurs ont noté que les excès de distribution occasionnaient le gaspillage par les oiseaux et impactaient négativement la performance des pondeuses. De plus, la distance entre les fermes et les habitations environnantes ou les fermes voisines avaient une influence positive significative sur l'ET. Les fermes étaient plus distantes les unes des autres (plus de $1,5 \mathrm{~km}$ ) que des maisons d'habitation (694,78 m) (tableau VI). Plus les exploitations sont éloignées les unes des autres, plus le risque de propagation de maladie est réduit et meilleure peut être la performance de production. Les données de l'étude étaient largement au-dessus de la distance minimale recommandée qui varie de 25 à $100 \mathrm{~m}$ entre une exploitation avicole et les maisons d'habitation (Ecale, 2014F).

Le prix des œufs a exercé une influence positive significative sur les niveaux d'ET des exploitations. Le prix constitue l'élément qui soustend l'engagement de tout exploitant. Statistiquement, la variation à la hausse d'une unité de prix contribuait à l'accroissement considérable des scores d'ET $(0,805)$. Ce résultat montre l'intérêt d'un prix sécurisé pour les aviculteurs. Les prix étant très fluctuants, les aviculteurs ont intérêt à passer des contrats de commercialisation avec leurs clients. Cette situation interpelle les pouvoirs publics béninois mais les structures étatiques en charge de l'accompagnement des producteurs, en l'occurrence le Carder, sont absentes sur le terrain, leur intervention se limitant généralement à la collecte de données. Plus de $86 \%$ des exploitants ont rapporté n'avoir reçu aucun soutien de l'Etat (tableau VII). Pourtant ce soutien, à travers les subventions aux agriculteurs, contribue à l'amélioration de la compétitivité (Siéwé et al, 2017). Outre la subvention, l'Etat devrait faciliter l'accès des producteurs au crédit notamment à travers les fonds de garantie. Au Bénin, la majorité des aviculteurs a rarement bénéficié d'un prêt pour démarrer son activité. Seulement $18,51 \%$ a pu obtenir un financement auprès de structures d'appui aux petites et moyennes entreprises (FAO, 2015). Une étude en 2007 de la Direction de l'élevage montre que seulement $38,3 \%$ d'aviculteurs bénéficient d'un crédit de la part des institutions de microfinance et quelques rares fois des banques (Guézodje, 2009).

Certaines variables exercent des effets négatifs significatifs sur les niveaux d'efficacité. L'effet négatif du niveau d'instruction de l'éleveur sur l'efficacité technique est significatif et peut se traduire par un moindre respect des itinéraires techniques (prophylaxie, horaires de distribution des aliments, renouvellement régulier de pédiluve...). Bien que son niveau d'instruction soit quasi égal à celui d'un propriétaire
(12,4 ans d'étude), en général, contrairement à ce dernier, il ne bénéficie pas de formations en aviculture (Batonon-Alavo et al., 2015).

De même, le poids des poules à l'entrée en ponte a eu une influence négative significative sur les niveaux d'efficacité. Le poids requis à l'entrée en ponte à partir de la dix-huitième semaine est de $1,5 \mathrm{~kg}$ (PACA, 2013). Le poids moyen obtenu dans notre étude de $1,51 \mathrm{~kg}$ était dans la norme, mais le quartile d'ordre 1 montre que le poids des poules entrées en ponte était de $1,48 \mathrm{~kg}$ chez $25 \%$ des éleveurs, alors que le quartile d'ordre 3 montre qu'il était de $1,6 \mathrm{~kg}$ chez $75 \%$ d'entre eux. Ces valeurs différentes de la norme peuvent expliquer l'influence négative du poids des futures pondeuses sur la productivité. Le surpoids peut provoquer la fatigue chez les jeunes pondeuses, ce qui peut affecter le rendement. Par ailleurs, avec un poids en dessous de la norme, les déficiences alimentaires se répercutent plus vite sur les performances de l'élevage (Hy-Line, 2013). Ces déficiences alimentaires légères provoquent généralement des problèmes de squelette et/ou de qualité de coquille. Les déficiences alimentaires importantes entraînent toujours des chutes de production notoires.

L'aviculture béninoise rencontre également des problèmes en termes de recrutement de personnes qualifiées ce qui a un effet pervers sur la performance de l'exploitation; les employés sont peu nombreux et très souvent recrutés sans formation préalable (Guézodje, 2009). Les rares personnes ayant suivi une formation dans le domaine préfèrent créer leur propre élevage ou travailler dans le secteur public.

En outre, il existe une corrélation négative entre les litiges avec le voisinage et la performance des exploitations. Les activités de production avicoles causent des nuisances environnementales, notamment des odeurs issues des déchets produits, qui sont à l'origine de conflits pouvant aller jusqu'à menacer la survie de l'exploitation.

Entre les dépenses de fidélisation des clients et les scores d'ET il y avait une forte relation négative. Les producteurs dépensaient en moyenne 64,11 FCFA par plateau d'œufs vendus. Cette dépense ne garantissait pas la fidélité des grossistes et semi-grossistes dans la durée. Ceux-ci à travers des comportements opportunistes s'approvisionnaient auprès de l'aviculteur du moment le plus offrant. La majorité des aviculteurs $(90 \%)$ reconnaissait avoir été régulièrement confrontée au non-respect des commandes passées avec les grossistes. Ces dépenses représentaient en moyenne plus de 2 FCFA par œuf avec un maximum de 6 FCFA. Or le revenu net par œuf était de 3,72 FCFA. Ces dépenses représentaient $53,7 \%$ du bénéfice net par œuf vendu. Ce résultat explique leur influence négative sur la performance. A l'inverse, Batonon-Alavo et al. (2015) montrent l'intérêt des pratiques de fidélisation en aval de la filière notamment pour la commercialisation des œufs. Les éleveurs entretiennent une relation de fidélité avec leurs clients grossistes à qui ils vendent de façon prioritaire en cas d'augmentation de la demande ou à paiement différé en cas de mévente.

\section{CONCLUSION}

Les exploitations enquêtées étaient en moyenne toutes efficaces techniquement. Cependant, les caractéristiques techniques des différentes souches élevées, l'impact d'une limitation des importations des produits avicoles sur la performance des aviculteurs et sur la fiscalité au Bénin, entre autres, enrichiraient la compréhension de l'efficacité technique et du fonctionnement de la filière avicole au Dahomey.

Les résultats nous permettent d'adresser des recommandations aux acteurs de la filière. Premièrement, les aviculteurs devraient faire les calculs technico-économiques afin d'avoir une bonne visibilité de l'évolution de leur activité, et adhérer aux associations d'aviculteurs pour bénéficier d'avantages relatifs (partage des expériences, formations, achats groupés de poussins, caution morale, etc.). 
Deuxièmement, l'Etat devrait soutenir l'accès des aviculteurs aux crédits via la subvention d'institutions de microfinances en mettant en œuvre « l'avi-finance » (financement avicole) pour soutenir la production avicole nationale, protéger les aviculteurs de la concurrence étrangère, et leur offrir la possibilité d'obtenir des produits avicoles à moindre coût. De plus, valoriser le protectionnisme à travers la mise en œuvre d'une réelle politique de réglementation tarifaire limiterait l'importation des produits avicoles. Troisièmement, les fournisseurs et fabricants d'intrants devraient respecter les formules des compositions alimentaires, et éviter la vente des produits avariés et des produits vétérinaires périmés dont l'utilisation met en péril toute l'activité avicole. Les vaccins étant généralement vendus pour 1000 poulets, ces produits vétérinaires devraient être aussi conditionnés pour des petits élevages de 250-500 poulets. Et quatrièmement, les organisations faîtières devraient organiser davantage de formations sur la maîtrise des itinéraires techniques de production avicole et sur la gestion économique des exploitations avicoles pour promouvoir leur durabilité.

\section{Remerciements}

Les auteurs remercient : l'Union européenne qui, dans le cadre de la mobilité Intra-ACP à travers le programme HAAGRIM à l'Université d'Abomey-Calavi, a financé ce travail ; l'UNAP-Bénin, notamment M. C. Azomahou pour avoir fourni des informations relatives à la filière avicole béninoise et avoir facilité la collecte de données ; les agents du Carder, en particulier M. Ephraïm, pour ses conseils et orientations pendant la phase de préenquête ; et les aviculteurs d'Abomey-Calavi, particulièrement Dr N. Akiyo et Mme A. Gnonlonfou pour leur collaboration et disponibilité.

\section{Déclaration des contributions des auteurs}

EBSP a participé à la conception, la planification, la collecte des données, la rédaction de la première version du manuscrit et aux échanges avec les relecteurs ; IM a contribué à la révision critique du manuscrit et a apporté des orientations lors de la phase de planification; IAL et TB ont participé à l'analyse des statistiques.

\section{REFERENCES}

Adebowale O., Adeyemo O., 2018. Characterization of bacterium types isolated from commercial laying hen farms in Ogun State Nigeria. Rev. Elev. Med. Vet. Pays Trop., 71 (3): 137-141, doi: 10.19182/remvt.31642

Aigner D., Lovell C.K., Schmidt P., 1977. formulation and estimation of stochastic frontier production function models. J. Econometr., 6 (1): 21-37, doi: 10.1016/0304 4076(77)90052-5

Albouchi L., Bacta M., Jacquet F., 2007. Estimation et décomposition de l'efficacité économique des zones irriguées pour mieux gérer les inefficacités existantes. In : Actes Sémin. Les instruments économiques et la modernisation des périmètres irrigués, 21-22 nov. 2005, Sousse, Tunisie (Ed. Bachta M.S.). Cirad, Montpellier, France, $19 \mathrm{p}$.

Amara N., Romain R., 2000. Mesure de l'efficacité technique : revue de la littérature. Centre de Recherche en économie agroalimentaire, Faculté des sciences de I'agriculture et de I'alimentation, Université Laval, Canada, 1-34 (Série Recherche ; 00.07)

Ambapour S., 2001. Estimation des frontières de production et mesures de I'efficacité technique. Doc Trav. 02/2001. BAMSI, Brazzaville, Congo, 27 p.

Atkinson E.S., Cornwell C., 1994. Estimation of output and input technical efficiency using a flexible functional form and panel data. Int. Econ. Rev., 35 (1): 245-255, doi: 10.2307/2527100

Atkinton K., Axtel B., Diop S., Divine E.E.G., Fellows P., Mchomvu H., Oti-Boateng P., et al., 2005. Créer et gérer une petite entreprise agroalimentaire. GRET, Paris, France, 274 p. (Coll. Réussir dans I'agroalimentaire)

Batonon-Alavo D.I., Bastianelli D., Chrysostome C.A.A.M., Duteurtre G., Lescoat P., 2015. Securing the flows of feed ingredient supplies and commercialization of products in the poultry sector: Case of the egg value chain in Benin. Rev. Elev. Med. Vet. Pays Trop., 68 (1): 3-18, doi: 10.19182/remvt.20571

Chemak F., Dhehibi B., 2010. Efficacité technique des exploitations en irrigué. Une approche paramétrique versus non paramétrique. New Medit., 9 (2) : $32-41$
Chrysostome C.A.A.M., Sodjinou E., 2005. Diagnostic de la filière des volailles et étude d'impacts de la phase 1 du Programme d'appui au développement de I'aviculture villageoise. Danish International Development Agency, Cotonou, Bénin

Coelli T.J., 1996. A guide to DEAP Version 2.1: A data envelopment analysis Working Papers No. 8/96. CEPA, University of New England, Australia, 50 p.

Coelli T., Fleming E., 2004. Diversification economies and specialization efficiencies in a mixed food and coffee small-holder farming system in Papua New Guinea. Agric. Econ., 31 (1): 229-239, doi: 10.1016/j.agecon.2004.09.010

Coelli T.J., Rao D.S., Battese G.E., 1998. An introduction to efficiency and productivity analysis. Kluwer Academic, Boston, USA, 275 p., doi: 10.1007/978-1-4615 5493-6

Debreu G., 1951. The coefficient of resource utilization. Econometrica, 19 (3): 273-292, doi: 10.2307/1906814

Ecale A., 2014. La réglementation des élevages. Distances d'implantation des installations d'élevage. Chambre d'agriculture des Deux-Sèvres, Vouillé, France, 3 p.

Fanou U., 2006. Revue du secteur avicole du Bénin. Division de la production et de la santé animale. FAO, Rome, Italie, 36 p.

FAO, 2015. Secteur avicole Bénin. Division de la production et de la santé animales de la FAO. Rev. Natl. Elev. (10) : 74

Farrell M.J., 1957. The measurement of productive efficiency. J. R. Stat. Soc. Ser. A, 120 (3): 253-290, doi: 10.2307/2343100

Fellows P., Franco E., Rios W., 1997. Starting a small food processing enterprise. Intermediate technology publication, London, UK, $128 \mathrm{p}$

Guézodje L., 2009. Des systèmes de production, des enjeux, des défis. Contraintes et défis de I'aviculture en Afrique de I'Ouest : cas du Bénin. Grain Sel (46-47) : 24-25

Hy-line, 2013. www.hyline.com

Koopmans T.C., Ed., 1951. Activity analysis of production and allocation. John Wiley, New York, USA, $430 \mathrm{p}$.

Kpenavoun Chogou S., Gandonou E., Fiogbe N., 2017. Mesure de l'efficacité technique des petits producteurs d'ananas au Bénin. Cah. Agric., 26 : 25004 doi : 10.1051/cagri/2017008

Labiyi I.A., Ayédèguè L., Yabi A.J., 2014. Analyse de l'efficacité économique d'allocation des ressources dans la production du soja au Benin. Lardes, Parakou, Bénin

Le Boterf G., 2004. Construire les compétences individuelles et collectives : la compétence n'est plus ce qu'elle était, $3^{\mathrm{e}}$ édn. Editions d'Organisation, Paris, France

Le Clainche E. 2008. Approche du concept de compétence. IFP Bretagne, Rennes, France, $5 \mathrm{p}$.

MAEP, 2014. Annuaire statistique de la Division de l'élevage. Bénin

MAEP, 2010. Plan stratégique de relance du secteur agricole. Bénin

Meeusen W., Van den Broeck J., 1977. Efficiency estimation from Cobb-Douglas production functions with composed error. Int. Econ. Rev., 18 (2): 435-444, doi: $10.2307 / 2525757$

NEPAD et FAO, 2005. Appui à la mise en œuvre du NEPAD-PDDAA, vol. 5 , Projet de développement de I'aviculture moderne en zones périurbaines et de I'aviculture traditionnelle en zones rurales. Gouvernement du Bénin

PACA, 2013. Conduite et gestion compétitive des élevages de volailles de chair et de ponte. Minader / Minepia, Cameroun

Panda R.C., 1996. Efficiency and productivity. The case of sericulture farms in Tamil Nadu. Ind. J. Agric. Econ., 51 (3)

Siéwé P.B., Kamajou F., Noula., 2017. Effets des innovations managériales sur la compétitivité agricole : cas des OP bénéficiaires du PACA dans la région de I'Ouest, Cameroun. Tropicultura, 35 (1) : 25-38

Sharma K.R., Leung P.S., Zaleski H.M., 1999. Technical, allocative and economic efficiencies in swine production in Hawaii: a comparison of parametric and non parametric approaches. Agric. Econ., 20: 23-35, doi: 10.1016/S01695150(98)00072-3

Sodjinou E., Aboh Boya A., 2009. Etude de la compétitivité des systèmes traditionnel et moderne d'élevage de la volaille au Bénin. INRA, Benin

Thanassoulis E., 2001. Introduction to the theory and application of data envelopment analysis: A foundation text with integrated software. Kluwer Academic, Boston, USA, 281 p., doi: 10.1007/978-1-4615-1407-7

Wilhelm M., 2014. Echantillonnage boule-de-neige. La méthode de sondage déterminé par les répondants. Office fédéral de la statistique, Neuchatel, Suisse $58 \mathrm{p}$.

Wittorski R., 1998. De la fabrication des compétences. Educ. Perm., 135 : 57-69 


\section{Summary}

Siéwé Pougoué E.B., Manu I., Labiyi Adédédji I., Bokossa T. Technical efficacy of laying hen farms in Southern Benin

The performance of national poultry farms has been a central issue in the context of massive imports of poultry products into Benin. Socioeconomic considerations have arisen in order to ensure a better allocation of productive resources. This study aimed to establish the performance level of laying hen farms in Southern Benin. Using the parametric approach, technical efficiency levels were estimated from a stochastic production frontier. We collected data on forty-five poultry farms with surveys conducted between August and September 2016. The analyses showed that the majority of the farms were performing well despite differences in efficiency between them. Human capital, labor, veterinary treatments were the main significant factors in the egg production process. The production function regression revealed that the low production of layers resulted more from technical shortcomings of producers $(84 \%)$ than from the inefficient allocation of resources $(16 \%)$. Differences in inefficiency were caused by socioeconomic factors including State support, the educational level, the poultry farmer's age, his/her skills, and poultry density. In conclusion, the poultry farms surveyed in Benin performed well but were weakened by external factors.

Keywords: poultry, layer chicken, efficiency, stochastic processes, livestock management, Benin

\section{Resumen}

Siéwé Pougoué E.B., Manu I., Labiyi Adédédji I., Bokossa T. Eficacia técnica de las granjas de gallinas ponedoras en el sur de Benin

El desempeño de las granjas avícolas nacionales es fundamental para el debate, en un contexto de importaciones masivas de productos avícolas a Benin. Surgen consideraciones socio-económicas para garantizar una mejor distribución de recursos productivos. Este estudio tuvo como objetivo establecer el nivel de rendimiento de las granjas de gallinas ponedoras en el sur de Benin. Utilizando el enfoque paramétrico, se estimaron los niveles de eficiencia técnica, a partir de una línea de producción estocástica. Los datos se recopilaron de cuarenta y cinco granjas avícolas con encuestas realizadas entre agosto y septiembre de 2016. Los análisis mostraron que la mayoría de estas granjas se desempeñaban bien, a pesar de diferencias de eficiencia entre ellas. El capital humano, el trabajo y los tratamientos veterinarios fueron los principales factores significativos en el proceso de producción de huevos. La regresión de la función de producción reveló que la baja producción de ponedoras resultó más de las deficiencias técnicas de los productores $(84 \%)$ que de una distribución ineficiente de recursos $(16 \%)$. Las diferencias en la ineficiencia fueron causadas por factores socioeconómicos, entre ellos el apoyo estatal, el nivel educativo, la edad del criador de aves, sus habilidades y la densidad de las aves. En conclusión, las granjas avícolas encuestadas en Benin obtuvieron buenos resultados, pero se vieron debilitadas por factores externos.

Palabras clave: aves de corral, gallina ponedora, eficacia, procesos estocásticos, manejo del ganado, Benin 